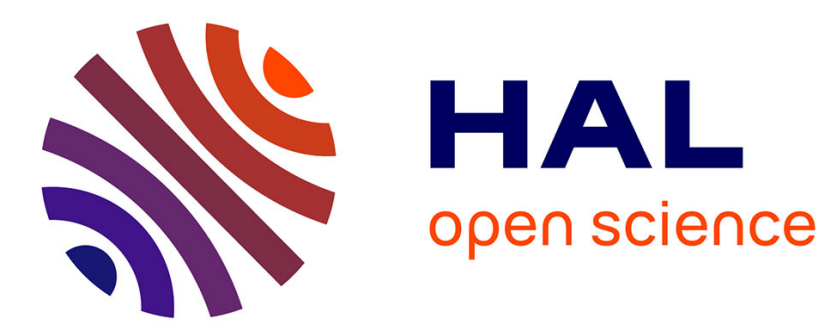

\title{
Assessment of the critical dimension prediction accuracy for the lumped parameter model for resist lithography
}

David Fuard, Patrick Schiavone

\section{To cite this version:}

David Fuard, Patrick Schiavone. Assessment of the critical dimension prediction accuracy for the lumped parameter model for resist lithography. Microelectronic Engineering, 2004, 73-74, pp.53-58. 10.1016/j.mee.2004.02.015 . hal-00021022

\section{HAL Id: hal-00021022 https://hal.science/hal-00021022}

Submitted on 16 Mar 2006

HAL is a multi-disciplinary open access archive for the deposit and dissemination of scientific research documents, whether they are published or not. The documents may come from teaching and research institutions in France or abroad, or from public or private research centers.
L'archive ouverte pluridisciplinaire HAL, est destinée au dépôt et à la diffusion de documents scientifiques de niveau recherche, publiés ou non, émanant des établissements d'enseignement et de recherche français ou étrangers, des laboratoires publics ou privés. 


\section{Assessment of the Critical Dimension (CD) prediction accuracy of the Lumped}

\section{Parameter Model (LPM) for resist lithography.}

D. Fuard, P. Schiavone

Laboratoire des Technologies de la Microélectronique, CNRS, c/o CEA Grenoble, 17 rue des Martyrs, 38054 GRENOBLE cedex 9, France

fuardda@chartreuse.cea.fr

\section{Keywords}

Lumped Parameter Model, simplified resist models, Bossung, Focus Exposure Matrix, Critical Dimension, CD prediction, simulation, lithography. 


\section{Abstract}

Lithography modelling is widely used to predict the Critical Dimensions (CD) of patterned features after lithographic processing. A lot of full and simplified resist models are available. Previous works on full resist models have shown that the numerous model parameters are very difficult to set, and often have a poor range of validity outside the dataset that have been used to generate them $[1,2]$. Simplified resist models are an alternative solution, easier to set, and they often provide a good simulation accuracy $[3,4]$. Among simplified models, LPM is widely used for CD predictions.

In this article, we study the CD prediction accuracy of the Lumped Parameter Model, using a comparison between experimental and simulated data. A systematic method is applied for LPM parameters extraction (contrast $\gamma$, and effective thickness $D_{\text {eff }}$ ). This assessment shows that a single parameters set giving reasonable accuracy is not found. Moreover, the critical analysis of the model parameters shows that these LPM parameters have a poor physical meaning. We also point out that there is a fundamental disagreement between the LPM theory and experiments. 


\section{INTRODUCTION}

The use of simulation for CD prediction is often mandatory to avoid heavy experimental work and to decrease the cost of $R \& D$. These simulations can be conducted either using full or simplified resist models. Full resist models are very difficult to tune and the large number of required parameters is a major source of simulation to experiment mismatch. In practice, they often provide a questionable reliability for $\mathrm{CD}$ prediction, despite the fact that they are based on fundamental physical considerations.

Simplified resist models represent an alternative to full resist models. They rely on simpler physical considerations and use fewer model parameters. Among all the models available for CD prediction, the Lumped Parameter Model (LPM) is widely used.[5] In this paper, the CD prediction of the LPM as well as the physical meaning of the model parameters are analysed. The LPM simulation results are compared with experimental data using a large number of illumination conditions, wavelengths, mask designs, and feature types.

\section{EXPERIMENTAL DATASETS}

2D and 1D features have been used for our comparison, as well as two different wavelengths (248nm and $193 \mathrm{~nm})$. The available datasets for LPM prediction accuracy are listed below, and the detailed experimental conditions are summarized in Table 1.

Contact holes@248nm:

The features were obtained using $0.45 \mu \mathrm{m}$ thick JSR M79Y resist, conventional illumination and $6 \%$ attenuated PSM. The nominal CD of the holes on the mask is 200 and $220 \mathrm{~nm}$, with different Space to Line ratios $\mathrm{L}: \mathrm{S}=1: 0.6$ and 1:2.2. A 248nm Nikon S204B Scanner (numerical aperture (NA) of 0.68 , and partial coherence $(\sigma)$ of 0.6 ) and a Hitachi Critical Dimension Scanning Electron Microscope (Hitachi S9300 SEMCD) were respectively used 
for the exposures and for $\mathrm{CD}$ measurements. No mask correction has been applied in the simulations.

\section{$\underline{\text { Lines@193nm: }}$}

The final features were obtained with $0.5 \mu \mathrm{m}$ thick Sumitomo PAR 707 resist on $75 \mathrm{~nm}$ thick anti reflective coating AR19. For the exposure, we used an ASML/900 193nm stepper with conventional illumination and binary masks. The nominal CD of the lines on the mask are $120 \mathrm{~nm}$ with a set of nested and isolated lines (line to space ratios $\mathrm{L}: \mathrm{S}=1: 1.5,1: 1.75,1: 2,1: 3$ and isolated), using $0.63 \mathrm{NA}$ and two different partial coherences of 0.6 and 0.85 . This provides a set of ten different settings on which the simulation is globally assessed.

\section{LPM DESCRIPTION AND CRITICAL DISCUSSION}

The Lumped Parameter Model (LPM) considered in this work is the enhanced LPM published by C. A. Mack in 1994 [5]. It takes as its input the aerial image of one or two-dimensional mask features and two lumped parameters. These parameters are the resist contrast $\gamma$ and effective thickness $D_{\text {eff }}$, which are used to compute the developed resist feature size. The LPM uses a simple approach relating the development time to the exposure dose, while the aerial image simulation is derived in a classical way. The development model relies on the assumption of a constant contrast. The model is also based on the hypothesis of a segmented development process, with two steps: a vertical development down to a depth $\mathrm{z}$, followed by a lateral development up to the position $\mathrm{x}$. The $\mathrm{CD}$ for a given exposure energy $E$ is found by solving equation 1 :

$$
\int_{0}^{C D / 2}\left(\frac{I\left(x^{\prime}\right)}{I(0)}\right)^{-\gamma} d x^{\prime}=\gamma \cdot D_{e f f}\left[\left(\frac{E}{E(0)}\right)^{\gamma}-1\right]
$$

where $\frac{I\left(x^{\prime}\right)}{I(0)}$ is the normalized image intensity and $E(0)$ is the dose to clear the resist.

More details on the derivation can be found in reference 5 . 


\subsection{Experimental CD behaviour through doses}

Previous works $[3,6]$ have shown that experimental Focus Exposure Matrix (FEM) data can be simply represented as an expansion of the feature dimension around the isofocal $\mathrm{CD}$. A representation (Figure 1) of the experimental CDs behaviour as a function of the aerial image intensity thresholds (or inverse of experimental dose [3]) for fixed defocus, gives an accurate insight into the experimental $\mathrm{CD}$ behaviour. This representation shows that all constant defocus curves are odd functions of intensity threshold. A major observation on the experimental $\mathrm{CD}$ behaviour is that the inflection points of each constant defocus curve are all located at the same CD position, namely the isofocal $C D$. (More precisely, all defocus curves cross at the same point, which is located at the isofocal $C D$ and at the isofocal intensity threshold). The following polynomial expression of experimental FEM can represent this fact $[3]:$

$$
C D_{\text {exp }}=\left[\sum_{k \in \square} a_{k} \cdot\left(f-f_{0}\right)^{2 k}\right] \cdot\left[\sum_{n \in \square} b_{n} \cdot\left(t-t_{0}\right)^{2 n+1}\right]+\text { isofocal } C D
$$

(where $f$ is the defocus, $f_{0}$ the defocus at best focus, $t$ the image intensity threshold, $t_{0}$ the image intensity threshold at isofocal CD. $f_{0}, t_{0}$, isofocal $C D, a_{k}$ and $a_{n}$ are constants. A good fitting expression is obtained using only $\mathrm{k}=[0,1,2]$ and $\mathrm{n}=[0,2] .[3])$ This expression takes into account that isofocal $C D$ is constant for $t=t_{0}$, and that all inflection points of defocus curves are located at the same isofocal $\mathrm{CD}$ for a given defocus $f$.

\subsection{The simulated CD behaviour for LPM}

Figure 2 uses the representation of the previous section (Figure 1) to illustrate the computed LPM CD behaviour as a function of the Log exposure dose, for various defocus. This plot shows that there is a fundamental disagreement between experimental FEM and the LPM theory, because LPM isofocal CD is no more at the same location than the inflection point of the curves. The mismatch between the isofocal $C D$ and the inflection point is a strong indication of the inability of LPM to provide accurate simulation of experimental data for 
which these two points always match. Since we deal with monotonic and continuous functions, the fact that the log exposure dose is plotted (whereas inverse of dose is plotted on Figure 1) does not change this conclusion.

\section{ASSESSMENT OF THE LPM CD PREDICTIONS CAPABILITIES}

\subsection{Method}

In this section, we will assess the LPM CD prediction accuracy. The experimental datasets (expressed as a function of experimental doses and defocus) are compared to Focus-Exposure Matrices computed using the LPM (expressed as a function of Log exposure doses and defocus). To compare the FEMs, the experimental doses must match the Log exposure doses for each Bossung curve. Since the experimental doses and the dose to clear of the resist are not exactly defined, we modified the original expression $\ln \left(\frac{E(x)}{E(0)}\right)$ of reference 5 into $\ln \left(\frac{d-\Delta E}{E(0)}\right)$, where $d$ represents the experimental dose, $E(0)$ represents the dose to clear and $\Delta E$ represents a potential dose offset. Thus, a general relation between the experimental dose $d$ and the log exposure dose $d_{L P M}$ can be written as $d=E(0) \cdot \exp \left(d_{L P M}\right)+\Delta E$, where $E(0)$ and $\Delta E$ are two adjustable parameters of the model. This does not change the basics of the Lumped Parameter Model but adds one more parameter to tune the model.

The LPM that we use here requires the determination of five resist parameters, which are assumed of general use for a given resist process (resist thickness, layer stack, baking and development conditions) and a given metrology procedure. These coefficients should be the same for all illumination conditions (all masks types, all features types, all optical settings, etc.) and this process can be represented by the expression:

$$
C D_{\text {simul }}=C D_{L P M}\left(\ln \left(\frac{d-\Delta E}{E(0)}\right), \gamma, D_{e f f}\right)+\Delta C D
$$


where: $C D_{L P M}$ is the CD provided by the LPM calculation at a given Log exposure dose. The contrast $\gamma$ and the effective thickness $D_{e f f}$ are parameters of the rough LPM. A fifth coefficient $\triangle C D$ is added as an additional degree of freedom accounting for a possible offset between experimental and simulated CDs. To find out these five coefficients, an optimisation procedure is performed using a Levenberg-Marquardt algorithm. The coefficients are extracted at the same time for all conditions or features for a given resist process. This way, we obtain two sets of LPM coefficients for each set of previously described $248 \mathrm{~nm}$ and $193 \mathrm{~nm}$ data.

\subsection{Results of LPM CD predictions}

The results of the model parameters determination are gathered in Table 2. Figure 3 and Figure 4 show respectively two examples of superimposition of the best simulated CD predictions on experimental data for both contact holes and lines respectively printed at $248 \mathrm{~nm}$ and $193 \mathrm{~nm}$. These figures illustrate that, with the LPM coefficients giving the best CD prediction, the accuracy can at the same time be good for some features (e.g. 120nm isolated lines@193nm on Figure 3b, or 220nm semi isolated contacts@248nm on Figure 4a) and can give poor match for others (e.g. 120nm nested lines (L:S=1:1.5) @ 193nm on Figure 3a, or 200nm semi isolated contacts @248nm on Figure 4b). This shows that for these sets of data, LPM does not provide very accurate results.

Moreover, Table 2 shows that the best CD prediction for lines can be obtained using several values of contrasts ranging from 1 to 2 . We also notice that the effective thickness $D_{\text {eff }}$ is always very low (almost 0 ). These two LPM parameters exhibit a poor physical meaning here in comparison with the $8 \pm 1$ experimentally measured contrast and the experimental $0.5 \mu \mathrm{m}$ resist thickness. For contacts, the contrast (10 to 15$)$ and effective thickness $D_{\text {eff }}(0.7$ to $0.9 \mu \mathrm{m}$ ) show values closer to real ones (contrast of $5 \pm 1$, experimentally measured, and resist thickness of $0.45 \mu \mathrm{m}$ on $0.05 \mu \mathrm{m}$ Bottom Anti-Reflective Coating). 


\section{CONCLUSION}

We have shown that the LPM simulated CDs do not follow the behaviour of experimental CD. The generated LPM Focus-Exposure Matrix are fundamentally different from the experimental reality. The assessment of CD prediction shows as well that LPM provides generally poor accuracy. In addition, the LPM coefficients set giving the best CD prediction could generally not provide information on the resist process because the values given by the model parameters have no realistic physical meaning (best fits are obtained for lines with low contrast $\gamma$ and zero effective thickness $D_{e f f}$ ). For these reasons, the LPM can hardly be considered as a reliable predictive model for $\mathrm{CD}$.

One explanation of the disappointing results provided by this widely used model can be the following: the basic LPM is a rather old model [5] and was performing well in a range of features sizes where resist diffusion was almost negligible. This is not the case anymore and the LPM, which is a development model, cannot account for that. Including diffusion effects into the model would certainly help the LPM to increase its accuracy. 


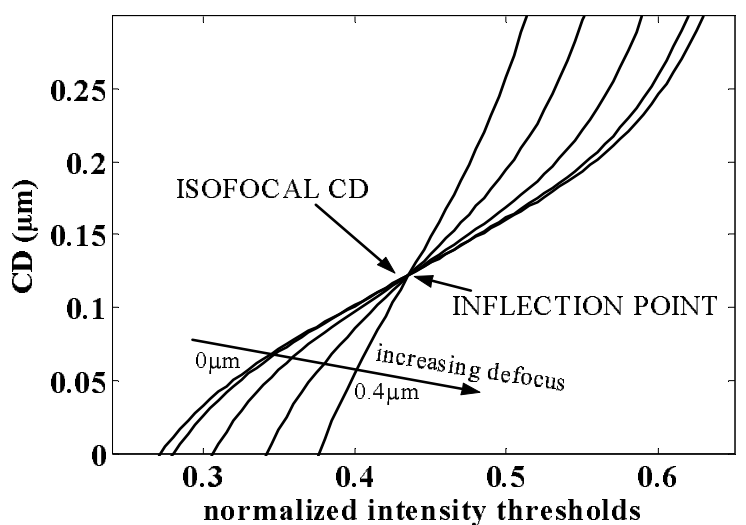

Figure 1

D. Fuard, "Lumped Parameters Model (LPM): Assessment of the Critical Dimensions (CD) prediction accuracy based on various experimental test cases." 


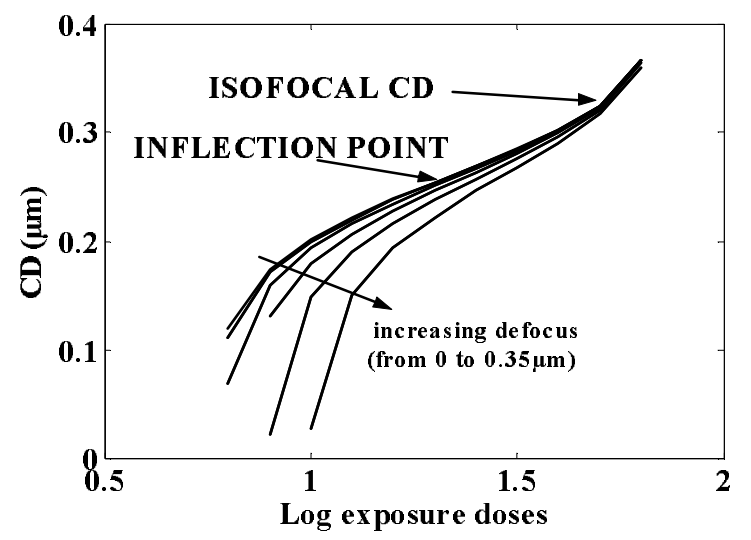

Figure 2 


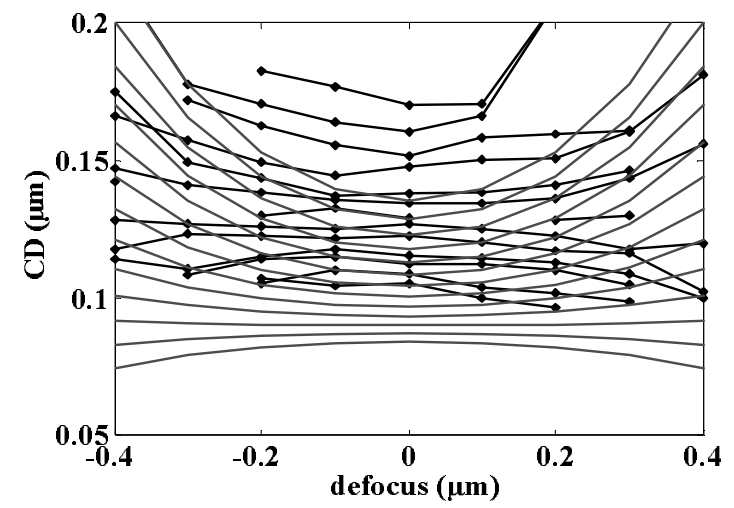

(a)

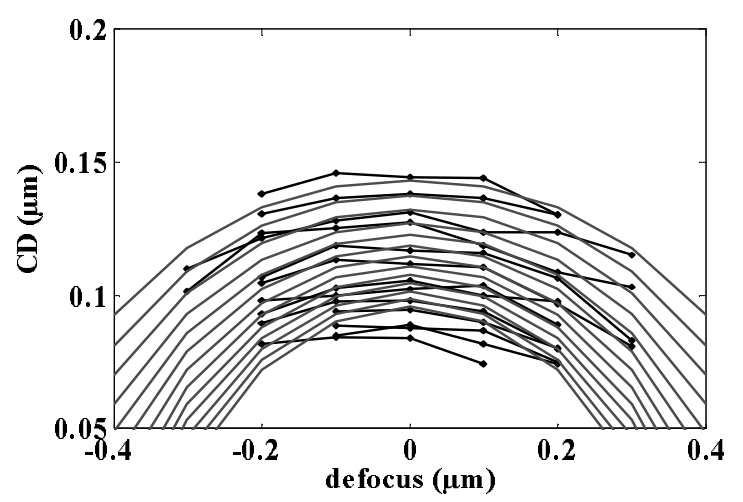

(b)

\section{Figure 3}




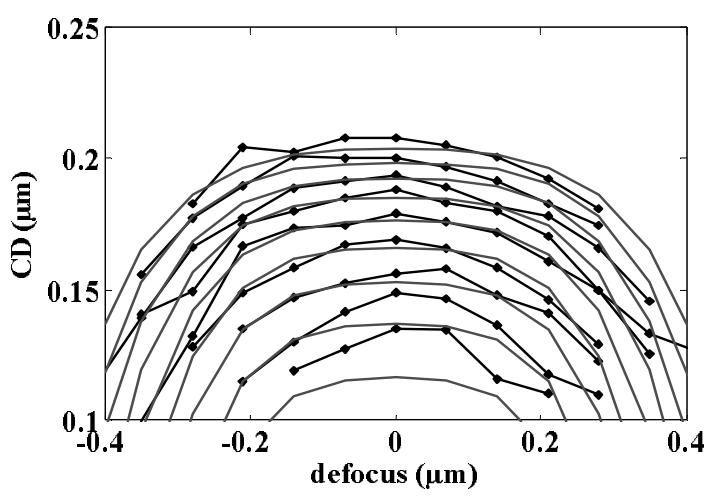

(a)

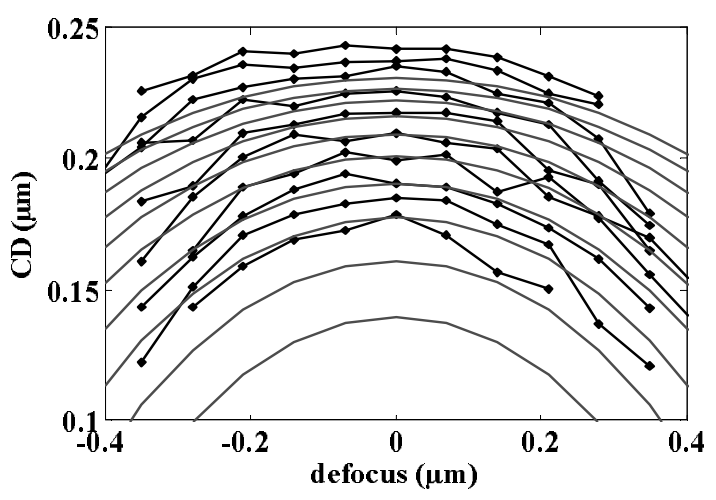

(b)

\section{Figure 4}




\begin{tabular}{l|c|c}
\hline \hline Exposure tool & ASML /900 & Nikon S204B Scanner \\
Wavelength & $193 \mathrm{~nm}$ & $248 \mathrm{~nm}$ \\
Illumination & Conventionnal & Conventionnal \\
Numerical Aperture & 0.63 & 0.68 \\
Partial coherence $\sigma$ & 0.6 and 0.85 & 0.6 \\
\hline Mask & Binary mask & $6 \%$ attenuated PSM \\
Features & 120 nm lines & 200 and $220 \mathrm{~nm}$ contacts \\
Line to Space ratio L:S & $1: 1.5,1: 1.75,1: 2$, & $1: 0.6,1: 2.2$ \\
& $1: 3$ and isolated & \\
\hline Resist & Sumitomo PAR 707 & JSR M79Y \\
Thickness & $0.5 \mu \mathrm{m}$ & $0.45 \mu \mathrm{m}$ \\
\hline \hline
\end{tabular}

Table 1

D. Fuard, "Lumped Parameters Model (LPM): Assessment of the Critical Dimensions (CD) prediction accuracy based on various experimental test cases." 


\begin{tabular}{|c|c|c|}
\hline & $\begin{array}{c}\text { 120nm lines @ 193nm } \\
\text { (Whole dataset) }\end{array}$ & $\begin{array}{c}200 \& \text { 220nm contacts } @ 248 \mathrm{~nm} \\
\text { (Whole dataset) }\end{array}$ \\
\hline resist contrast $\gamma$ & 1 to 2 & 10 to 15 \\
\hline effective thickness $D_{\text {eff }}$ & $<0.2 \mu \mathrm{m}$ & 0.7 to $0.9 \mu \mathrm{m}$ \\
\hline$E(0)$ coefficient & $4.05 \mathrm{~mJ} . \mathrm{cm}^{-2}$ & $8.2 \mathrm{~mJ} . \mathrm{cm}^{-2}$ \\
\hline$\Delta E$ coefficient & $-0.05 \mathrm{~mJ} . \mathrm{cm}^{-2}$ & $-3 \mathrm{~mJ} . \mathrm{cm}^{-2}$ \\
\hline$\Delta \mathrm{CD}$ & $0 \mathrm{~nm}$ & $5 \mathrm{~nm}$ \\
\hline CD prediction accuracy & $8.9 \%$ & $4.9 \%$ \\
\hline
\end{tabular}

Table 2

D. Fuard, "Lumped Parameters Model (LPM): Assessment of the Critical Dimensions (CD) prediction accuracy based on various experimental test cases." 


\section{Figure caption}

Figure 1: $\mathrm{CD}$ as a function of normalized intensity threshold for various defocus (ranging from 0 (best focus) to $0.3 \mu \mathrm{m}$ ), for $120 \mathrm{~nm}$ nested lines ( $L: S=1: 1.5$ ), $\lambda=193 \mathrm{~nm}$, $\mathrm{NA}=0.63$ and $\sigma=0.6$.

Figure 2: simulated $\mathrm{CD}$ as a function of the log exposure doses for various defocus (ranging from 0 (best focus) to $0.42 \mu \mathrm{m}$ ), for $200 \mathrm{~nm}$ dense contacts (pitch $=320 \mathrm{~nm}$ ), $\lambda=248 \mathrm{~nm}$, $\gamma=6, D_{\text {eff }}=0.2, E(0)=8.2, \Delta E=-3$ and $\Delta C D=5 \mathrm{~nm}$.

Figure 3: Superimposition of simulated LPM FEM (dashed lines, using the best parameters extracted from whole dataset) on experimental data (solid lines + diamonds) for $\lambda=193 \mathrm{~nm}, 120 \mathrm{~nm}$ lines with $\mathrm{L}: \mathrm{S}=1: 1.5$ (a) and with isolated lines (b).

Figure 4: Superimposition of simulated LPM FEM (dashed lines, using the best parameters extracted from whole dataset) on experimental data (solid lines + diamonds) for $\lambda=248 \mathrm{~nm}$, using $200 \mathrm{~nm}$ contact holes and $640 \mathrm{~nm}$ pitch (a) and $220 \mathrm{~nm}$ contact holes and $720 \mathrm{~nm}$ pitch (b). 


\section{Table caption}

Table 1: Summary of the experimental datasets.

Table 2: LPM parameters giving the best CD prediction for the whole dataset. 


\section{References}

[1] D. Kang, E. K. Pavelchek \& C. Swible-Keane, Proc. SPIE Vol. 3678, pp. 877-890 (1999).

[2] A. Erdmann, W. Henke, S. Robertson, E. Richter, B. Tollkühn, W. Hoppe, Proc. SPIE Vol. 4404, pp. 99-110 (2001).

[3] D. Fuard, M. Besacier and P. Schiavone, Proc. SPIE Vol. 4691, pp. 1266-1277 (2002).

[4] D. Fuard, M. Besacier and P. Schiavone, Proc. SPIE Vol. 5040, pp. 1536-1543 (2003).

[5] C. A. Mack, Proc. SPIE Vol. 2197, pp. 501-510(1994).

[6] C. P. Ausschnitt, Proc. SPIE Vol. 1088, pp. 115-123 (1989). 